\title{
Construcción y evaluación psicométrica de una escala para pesquisar factores vinculados al comportamiento suicida en adolescentes chilenos
}

Construction and psychometric assessment of a scale designed to
examine factors linkes to suicidal behavior in chilean adolescents

Recibido: septiembre 10 de 2009 | Revisado: mayo 29 de 2010 | Aceptado: junio 24 de 2010

\author{
AlFONSO URZÚA M.* \\ Universidad Católica del Norte, Antofagasta, Chile \\ AleJANDRA CAQUEO-URÍZAR ** \\ Universidad de Tarapacá, Arica, Chile
}

SICI: 1657-9267(201112)10:3<721:CEFVCS>2.3.TX;2-N

Para citar este artículo. Urzúa M., A. \& CaqueoUrizar, A. (2011). Construcción y evaluación psicométrica de una escala para pesquisar factores vinculados al comportamiento suicida en adolescentes chilenos. Universitas Psychologica, 10 (3), 721-734.

Escuela de Psicología, Avda. Angamos 0610, 1270709 Antofagasta, Chile. Teléfono: $(+55)$ 355843.E-mail: alurzua@ucn.cl

** Carrera de Psicología, Cardenal Caro 348, Arica, Chile. Teléfono: (+55) 205813.E-mail: acaqueo@ uta.cl
RESUMEN

Se evalúan las propiedades de un instrumento que pesquisa factores de riesgo del intento de suicidio, en 500 alumnos de enseñanza secundaria (media de edad de 16.5 años). La solución de cuatro factores propuesta (conductas suicidas, satisfacción, sentido de vida y afecto negativo) explica un $58 \%$ de la varianza. La consistencia interna de los factores y de la escala total es buena (alfa de Cronbach de 0.95 para la escala total). Los análisis de regresión sugieren que la mejor variable predictiva para la conducta "intento de suicidio", corresponde a conductas suicidas (ideación y planificación suicida). La escala posee propiedades psicométricas adecuadas para evaluar riesgo de intento suicida, discriminando entre aquellos que han realizado intentos de suicidio de aquellos que no lo han hecho.

Palabras clave autores

Suicidio, riesgo, adolescencia.

Palabras clave descriptores

Investigación cuantitativa, psicometría, construcción de pruebas psicológicas.

\section{A B S T R A C T}

It is assessed the properties of an instrument to detect risk factors for the attempt of committing suicide in 500 students attending high school (mean age 16.5 years). Proposed four-factor solution (suicidal behavior, satisfaction, sense of life, and negative affection) accounts for a $58 \%$ of the variance. Internal consistency for the factors and for the total scale is good (Cronbach's Alpha is 0.95 for the total scale). Regression analyses suggest that the best predictive variable for the "attempt-of-committing-suicide" behavior corresponds to suicidal behaviors (suicidal ideation and planning). The scale shows suitable psychometric properties to assess the risk for the attempt of committing suicide, differentiating between those who have made attempts of committing suicide and those who do not.

Key words authors

Suicide, risk, adolescence.

Key words plus

Quantitative research, psychometry, building of psychological tests. 
El suicidio ha llegado a convertirse en la segunda o tercera causa de muerte en jóvenes de 15 a 19 años de edad, observándose un significativo incremento de este fenómeno desde 1950 hasta la fecha, entre los adolescentes y adultos jóvenes de la población europea (Larraguibel, González, Martínez \& Valenzuela, 2000) y en los distintos países que componen las Américas y Asia (Cendales, Vanegas, Fierro, Córdoba \& Olarte, 2007; Hidaka et al., 2008; Li, Phillips, Zhang, Xu \& Yang, 2008; Puentes, López \& Martínez, 2004; Yunes \& Zubarew, 1999).

En Chile, las tasas de suicidio han tenido un aumento gradual en los últimos años, a excepción de los años 1992-1993, reportándose el año 2003 una tasa de 17.8 suicidios por 100.000 habitantes para los hombres y de 3.1 para las mujeres (World Health Organization [WHO], 2008). En los adolescentes, las tasas han experimentado un leve aumento durante el periodo 1995-1996. En este último año, la tasa de suicidio en la población general fue de 6.35 y de 2.87 para población adolescente (Valdivia, Ebner, Fierro, Fajardo \& Miranda, 2001). Dentro de las razones que pretenden dar cuenta de esto se encuentran: una mayor predisposición a desarrollar precozmente trastornos del ánimo (Yunes \& Rajs, 1994) y el incremento de las tasas de divorcio en las últimas décadas, lo que tiene como consecuencia, la mayoría de las veces, disfunción familiar (Birmaher, Ryan \& Williamson, 1996).

Los intentos de suicidio son un problema significativo en diversos países. Los estudios epidemiológicos revelan que entre un 3 y $5 \%$ de la población ha tenido un intento suicida (Valtonen, Suominen, Partonen, Ostamo \& Lönnqvist, 2006). Dicha cifra es especialmente importante dado que un número significativos de los adolescentes que consuman el suicidio, han presentado intentos o ideaciones previas (Valdivia et al., 2001).

Existe una serie de factores asociados al intento suicida, entre ellos está la morbilidad psiquiátrica; generalmente, la presencia de síntomas psiquiátricos (somatización, obsesión, depresión, ansiedad, hostilidad, fobia, paranoia, entre otros) tiene una relación directa con el riesgo de suicidio (Hidaka et al., 2008; Ono et al., 2008; Soo Kim \& Sil Kim,
2008). Diferentes estudios han puesto en evidencia una estrecha relación entre depresión y conducta suicida (Birmaher et al., 1996; Brent, Perper \& Moritz, 1993; Fleming, 2007; Harrington, Bredekamp \& Groothues, 1994; WHO, 2008), siendo este trastorno psiquiátrico el más prevalente tanto en el suicidio como en el intento de suicidio. En cuanto a la ansiedad, se ha encontrado que, considerada como rasgo, parece ser relativamente independiente de la depresión como factor de riesgo en la conducta suicida (Krysinska \& De Leo, 2008). Por otro lado, los trastornos de ansiedad podrían tener relación directa con el comportamiento suicida, es posible que el efecto que produce la angustia relacionada con los síntomas de ansiedad, pueda, de manera independiente, conducir al comportamiento suicida. También, los trastornos de ansiedad pueden ejercer un efecto indirecto, aumentando la probabilidad de una tercera variable (depresión, empleo de sustancias) que puede conducir al comportamiento suicida (Bolton, Cox, Afifi, Enns, Bienvenu \& Sareen, 2008). En la infancia, se plantea que la ansiedad agregada a un cuadro clínico de ideación suicida, crea un particular sufrimiento al estado psicopatológico infantil, llegando a ser un amplificador en el desarrollo y/o manutención de la ideación suicida (Larraguibel et al., 2000).

Otro factor de riesgo significativo se refiere a los problemas conductuales y de adaptación (Hawton \& Fagg, 1992; Minoletti \& López, 1999), asociados con conducta agresiva, conflictos legales, conducta sexual promiscua, alta impulsividad, abuso de sustancias y estatus socioeconómico. El abuso de sustancias se ha vinculado con la repetición de intentos de suicidio y con el suicidio completado (Hollis, 1996); además, junto con el estatus socioeconómico, es relevante para la comprensión del intento de suicidio, debido a que estos factores se asocian con el aumento de la posibilidad de formular ideas de suicidio y, a la vez, pueden progresar para franquear el intento de suicidio (Wong \& Stewart, 2007). Por otro lado, individuos con tendencia neurótica o baja en los niveles de extraversión, tienen menor probabilidad de buscar ayuda, y tienden a interpretar negativamente los elementos de su entorno, por lo tanto estarían predispuestos a la desesperación, la 
depresión y el suicidio (Shur-Fen Gau et al., 2008; Soo Kim \& Sil Kim, 2008).

Los sucesos vitales negativos en la niñez y la disfunción familiar se perfilan también como factores de riesgo importante. Dentro de los primeros, se encuentran: pérdidas a temprana edad, rechazo, problemas económicos, entre otros (Apter et al., 1995). La tensión familiar ha sido asociada directa o indirectamente con los comportamientos de riesgo del suicidio adolescente (Randell et al., 2006), donde la ausencia de calidez familiar, la falta de comunicación con los padres y la discordia familiar, hacen que existan escasas oportunidades para el aprendizaje de resolución de problemas, creando además un ambiente donde al adolescente le falta el soporte necesario para contrarrestar los efectos de eventos vitales estresantes (Larraguibel et al., 2000). Los adolescentes que intentan suicidarse, provienen de familias considerablemente más disfuncionales que aquellos adolescentes que no lo intentan; la influencia que puede tener una disfunción familiar sobre los comportamientos, puede prevalecer por sobre otras variables, como la depresión, la cual puede ser producto de la disfunción familiar percibida (Shur-Fen Gau et al., 2008; Silvikena \& Kvernmob, 2007; Soo Kim \& Sil Kim, 2008). Dentro del ámbito familiar, el estilo de crianza al igual que el funcionamiento familiar, son potentes predictores del riesgo de suicidio. Cuando los padres muestran un cuidado y afecto adecuado, el riesgo de suicidio es menor (Randell et al., 2006); en cambio, cuando los padres son percibidos como sobreprotectores o autoritarios, los riesgos de suicidio aumentan (Shur-Fen Gau et al., 2008); sin embargo, respecto de esto último, hay diferencias importantes que varían según la cultura. El estilo de educación autoritario se asocia de manera negativa con el logro académico entre estudiantes europeos y americanos, pero tiene una relación alta con el logro académico entre estudiantes chinos. Aunque unos argumenten que el estilo de educación autoritario lleva un significado negativo en el contexto occidental, esto no es el caso en la sociedad asiática (Shur-Fen Gau et al., 2008).

Por otro lado, considerando la variable sexo, los suicidios completados tienden a ser más comunes en hombres, en cambio en las mujeres es más prevalente el intento suicida (Randell et al., 2006; Wong \& Stewart, 2007). En general los estudios plantean una proporción de tres a un suicidio a favor de los hombres, sin embargo, se observan diferencias interesantes en algunos países como China, donde la tasa de suicidio es $25 \%$ más alta en mujeres que en hombres, mayoritariamente de poblaciones rurales (Li, Phillips, Zhang, Xu \& Yang, 2008; Phillips, Li \& Zhang, 2002) o en India donde la tasa es similar entre hombres y mujeres (Mayer \& Ziaian, 2002).

Existe evidencia de algunas diferencias entre los factores de riesgo en países desarrollados y los que están en vías de desarrollo; así, ser mujer, vivir en un área rural y mantener creencias religiosas que rechacen el suicidio, puede ser más relevantes como factor protector en países en vías de desarrollo. Por otro lado, ser soltero o tener antecedentes psiquiátricos parece tener menos peso como factor de riesgo en países en vías de desarrollo, por ser menos significativo en este tipo de países (Vijayakumar, John, Pirkis \& Whiteford, 2005).

Por último, considerando la edad de los adolescentes, se plantea que los suicidios son escasos en menores entre 10 y 14 años, siendo más común entre jóvenes de 15 a 19 años. Esto puede deberse a que los primeros tienen una menor exposición a factores de riesgo, presentan menos probabilidades de padecer algún trastorno del ánimo y que los factores madurativos comienzan a jugar un rol importante en los mayores: la capacidad de planear y llevar a cabo un acto suicida requiere de un nivel de maduración no alcanzado por niños o adolescentes tempranos (Larraguibel et al., 2000).

Por la relevancia de este tema para la salud pública y la salud mental en Chile, es que se propone un instrumento de corta aplicación, elaborado desde los constructos y vivencias actuales vinculadas al suicidio, que pueda ser utilizado como una herramienta de corte preventivo en establecimientos educacionales, posibilitando la pesquisa precoz y la intervención temprana en los adolescentes en riesgo.

En este contexto, el objetivo de esta investigación es evaluar las propiedades psicométricas 
de este instrumento de tamizaje y, paralelamente, describir cómo se comportan estos factores de riesgo, en una muestra de adolescentes chilenos.

\section{Método}

\section{Participantes}

La muestra para la aplicación piloto estuvo constituida por 60 alumnos, con similar porcentaje entre hombres y mujeres, que cursaban tercero o cuarto medio de un establecimiento municipalizado de enseñanza media de la ciudad de Antofagasta.

La muestra final estuvo conformada por 500 alumnos, de los cuales 199 (39.8\%) fueron hombres y 301 (60.2 \%) mujeres, alumnos de primero a cuarto medio de Establecimientos de Enseñanza Secundaria Municipalizada de la ciudad de Antofagasta. La edad promedio de los participantes correspondió para ambos sexos a 16.4 años $(D E=$ 1.0), con una edad mínima de 14 años para hombres y mujeres, y una edad máxima de 19 años para las mujeres y de 20 para los hombres.

En relación con el nivel de estudios, el mayor porcentaje se encontraba cursando tercero medio, con un $56.8 \%$, seguido de segundo medio (23\%), cuarto medio (16\%) y primero medio $(4.2 \%)$.

\section{Instrumento}

Con el objetivo de evaluar los factores relacionados con el intento de suicidio, se diseñó una primera escala compuesta por 82 reactivos, la cual incorporó variables que la literatura menciona como vinculadas a la presencia de conductas suicidas y, por otra parte, elementos recogidos en los grupos de discusión realizados con jóvenes, a saber: presencia de sintomatología depresiva, conductas suicidas, sintomatología ansiosa, satisfacción con la vida, autoestima y apoyo social.

Esta primera versión fue aplicada a la muestra piloto de 60 alumnos de enseñanza secundaria.

Con base en los análisis de consistencia interna, se eliminaron 22 reactivos que tuvieron un valor inferior a 0.5 en la correlación ítem-test. Los reactivos restantes fueron sometidos a un análisis factorial exploratorio y a una discusión teórica que permitió agrupar los ítems en cuatro factores, siendo estos: a) Conductas Suicidas, compuesto por 17 aseveraciones que evalúan los componentes: deseo de morir, representaciones suicidas, ideas suicidas y amenaza suicida, b) Satisfacción, que a través de 8 reactivos evalúa el grado de satisfacción consigo mismo y con la vida, c) Afecto Negativo, que a través de 5 reactivos evalúa la presencia de sintomatología asociada fundamentalmente a la depresión y d) Sentido de Vida, que igualmente a través de 5 reactivos evalúa aspectos vinculados a la autoestima y a la proyección con la vida. La distribución teórica de los factores puede ser revisada en el Anexo 1.

Se incluyó además un reactivo que inquiere, específicamente, sobre si se ha realizado un intento de suicidio.

Por último, la escala queda compuesta por 36 aseveraciones, en formato Likert, donde cada uno de los participantes debe expresar su grado de acuerdo o desacuerdo con cada una de las frases propuestas, en una medida de 1 a 4 , donde 1 corresponde a la categoría completamente en desacuerdo y el 4 a completamente de acuerdo. Para el análisis por factor, se invierte el puntaje de las preguntas que implican estar de acuerdo con una aseveración de valencia negativa, siendo los resultados interpretables de manera que a menor puntaje, mayor riesgo. El valor de cada dimensión es obtenido a través del promedio simple de las preguntas que la comprenden. Se calcula además un puntaje total equivalente el promedio de las cuatro dimensiones.

\section{Procedimiento}

Los participantes en el estudio fueron estudiantes de primero a cuarto año de enseñanza secundaria, de establecimientos educacionales municipalizados de la ciudad de Antofagasta, quienes fueron evaluados, previo consentimiento, en horas de consejo de curso, en distintos establecimientos. A estos se les explicó el objeto de estudio y se ejemplificó la manera de responder. Cada participante completó individualmente la escala, con un tiempo promedio de ejecución de 20 minutos. Una vez recolectadas 
las encuestas, fueron ingresadas a una base de datos en SPSS 14, para su posterior análisis estadístico.

\section{Análisis estadísticos}

Para evaluar la consistencia interna se utilizó el estadístico alfa de Cronbach. Posteriormente, se realizaron análisis factoriales de tipo exploratorio a través del método de componentes principales con rotación VARIMAX y confirmatorio, utilizando el programa Lisrel 8.3.

Como un indicador de la capacidad del instrumento para discriminar entre grupos con mayor o menor riesgo, se analizó la diferencia de medias en todas las dimensiones, entre aquellos que declararon haber realizado un intento de suicidio y aquellos que no, a través de la prueba $t$ para grupos independientes. A fin de evaluar la relación existente entre las dimensiones y las dimensiones con la escala total, se utilizó el coeficiente de correlación de Pearson.

Por último, a fin de analizar el valor predictivo de cada uno de los factores ante la conducta "intento de suicidio", se realizaron regresiones lineales.

\section{Resultados}

\section{Consistencia interna}

Se encuentran valores superiores a 0.9 para la escala total (0.954), factor Satisfacción (0.907) y Conductas Suicidas (0.947). El factor Afecto Ne- gativo reportó un alfa de 0.775 y el de Sentido de Vida, un alfa de 0.726 .

\section{Análisis de ítems}

La Tabla 1 muestra las correlaciones obtenidas para cada pregunta, en relación con su dimensión y con la escala total, así como la distribución porcentual de las respuestas en cada una de las alternativas.

En general, las correlaciones del ítem con su dimensión menos el ítem, son superiores a 0.45, encontrándose los valores para la dimensión Conducta Suicida entre 0.58 y 0.81 , entre 0.59 y 0.79 para Satisfacción, entre 0.46 y 0.56 para Sentido de Vida y entre 0.52 y 0.60 para Afecto Negativo. Las correlaciones ítem-escala total oscilaron entre 0.36 y 0.76 .

El promedio de porcentajes de respuestas no contestadas (perdidas) fue cercano al $1 \%$. Una pregunta alcanzó el 2 \% (sobre Objetivos de Vida), sin embargo, este porcentaje sigue siendo bajo. Se observa que la distribución de las respuestas tiende a centrarse en los puntajes altos, especialmente en aquellas vinculadas a Conductas Suicidas, donde el porcentaje promedio de respuestas en el puntaje mayor, es superior a un $60 \%$.

\section{Análisis Factorial}

Tanto el estadístico de adecuación muestral KMO (0.953) como la prueba de esfericidad de Bartlett $\left(\chi^{2(595)}=7696.674 ; p<0.001\right)$ indican la pertinencia de la realización de un análisis factorial.

TABLA 1

Análisis de ítems: correlación y efectos suelo y techo

\begin{tabular}{|c|c|c|c|c|c|c|c|}
\hline & \multicolumn{2}{|c|}{$\begin{array}{l}\text { Correlación } \\
\text { ítem }\end{array}$} & \multicolumn{5}{|c|}{$\begin{array}{c}\text { Distribución porcentual por } \\
\text { respuesta }\end{array}$} \\
\hline & Dim. & Total & $\% 1$ & $\% 2$ & $\% 3$ & $\% 4$ & Per. \\
\hline La idea de quitarme la vida me viene repetidamente a la cabeza. & 0.712 & 0.656 & 13.0 & 9.6 & 17.8 & 58.6 & 1.0 \\
\hline Creo que no merece la pena seguir viviendo. & 0.626 & 0.621 & 8.2 & 12.2 & 10.8 & 66.8 & 2.0 \\
\hline Algunas veces me he imaginado a mí mismo sin vida. & 0.582 & 0.548 & 17.4 & 13.8 & 19.4 & 48.6 & 0.8 \\
\hline He pensado en mi propio suicidio. & 0.756 & 0.727 & 10.8 & 10.8 & 15.0 & 61.4 & 2.0 \\
\hline
\end{tabular}




\begin{tabular}{|c|c|c|c|c|c|c|c|}
\hline & \multicolumn{2}{|c|}{$\begin{array}{l}\text { Correlación } \\
\text { item }\end{array}$} & \multicolumn{5}{|c|}{$\begin{array}{c}\text { Distribución porcentual por } \\
\text { respuesta }\end{array}$} \\
\hline & Dim. & Total & $\% 1$ & $\% 2$ & $\% 3$ & $\% 4$ & Per. \\
\hline Le he comentado a mis amigos que me gustaría quitarme la vida. & 0.622 & 0.538 & 8.6 & 5.2 & 11.2 & 73.8 & 1.2 \\
\hline Si me quisiera suicidar, tengo claro cómo lo haría. & 0.658 & 0.582 & 13.6 & 6.0 & 11.8 & 67.6 & 1.0 \\
\hline He simulado mentalmente mi suicidio. & 0.746 & 0.667 & 10.0 & 8.8 & 15.2 & 65.2 & 0.8 \\
\hline Sé con claridad cómo poder suicidarme sin fallar. & 0.632 & 0.585 & 11.2 & 6.4 & 11.0 & 70.8 & 0.6 \\
\hline Le he dicho a alguien que ya no quiero seguir viviendo. & 0.688 & 0.617 & 12.8 & 6.8 & 12.2 & 67.6 & 0.6 \\
\hline Tengo planificada la forma como podría quitarme la vida. & 0.749 & 0.684 & 6.6 & 5.8 & 11.0 & 76.0 & 0.6 \\
\hline A veces he tenido la fantasía de verme ahorcado. & 0.692 & 0.599 & 10.6 & 6.4 & 12.4 & 69.8 & 0.8 \\
\hline No sé cómo, pero me quiero quitar la vida. & 0.764 & 0.724 & 6.0 & 6.8 & 12.0 & 73.2 & 2.0 \\
\hline A veces pienso en suicidarme de cualquier manera... & 0.794 & 0.708 & 7.6 & 4.8 & 10.8 & 76.4 & 0.4 \\
\hline Quisiera morirme. & 0.806 & 0.759 & 9.0 & 7.8 & 11.8 & 70.4 & 1.0 \\
\hline Siento que la vida tal como la vivo no vale la pena. & 0.701 & 0.758 & 10.4 & 13.4 & 16.8 & 58.8 & 0.6 \\
\hline A veces me gustaría estar muerto y lejos de todo. & 0.736 & 0.709 & 17.2 & 9.4 & 15.2 & 58.0 & 0.2 \\
\hline Estoy aburrido de vivir. & 0.673 & 0.652 & 9.4 & 14.0 & 16.0 & 58.6 & 2.0 \\
\hline En general, estoy satisfecho conmigo mismo. & 0.618 & 0.536 & 10.2 & 24.2 & 39.4 & 25.4 & 0.8 \\
\hline Me siento orgulloso/a de quién soy y la vida que llevo. & 0.757 & 0.700 & 8.2 & 18.6 & 33.4 & 38.0 & 1.8 \\
\hline Estoy contento conmigo mismo. & 0.788 & 0.709 & 9.2 & 19.2 & 37.0 & 33.4 & 1.2 \\
\hline Estoy conforme con mi vida actual. & 0.761 & 0.719 & 11.6 & 19.8 & 35.2 & 31.6 & 1.8 \\
\hline Las condiciones de mi vida son excelentes. & 0.648 & 0.579 & 18.0 & 24.8 & 35.8 & 20.0 & 1.4 \\
\hline Estoy satisfecho con mi vida. & 0.784 & 0.717 & 11.6 & 22.2 & 30.4 & 35.0 & 0.8 \\
\hline Hasta el momento, he conseguido las cosas. & 0.701 & 0.591 & 13.4 & 23.8 & 35.6 & 26.6 & 0.6 \\
\hline Si pudiera volver a vivir mi vida, no cambiaría casi nada. & 0.585 & 0.524 & 30.4 & 22.4 & 23.6 & 23.4 & 0.2 \\
\hline Creo que soy importante para otros. & 0.456 & 0.452 & 6.4 & 18.2 & 34.6 & 39.8 & 1.0 \\
\hline Creo que tengo muchos motivos para enorgullecerme. & 0.448 & 0.467 & 6.8 & 20.8 & 36.8 & 34.4 & 1.2 \\
\hline Tengo claro qué es lo que intento conseguir en la vida. & 0.560 & 0.514 & 6.0 & 17.0 & 30.2 & 46.2 & 0.6 \\
\hline Tengo gente a mi alrededor que entiende mis puntos. & 0.454 & 0.423 & 11.1 & 19.0 & 34.2 & 34.8 & 1.0 \\
\hline Tengo clara la dirección y el objetivo de mi vida. & 0.516 & 0.549 & 8.8 & 17.4 & 35.8 & 35.0 & 3.0 \\
\hline A veces pienso que no sirvo para nada. & 0.604 & 0.561 & 23.6 & 21.6 & 18.4 & 35.2 & 1.2 \\
\hline Me he sentido solo. & 0.545 & 0.396 & 38.8 & 21.2 & 19.2 & 19.6 & 1.2 \\
\hline Me inclino a pensar que, en conjunto, soy un fracaso. & 0.562 & 0.646 & 9.6 & 14.0 & 19.6 & 55.0 & 0.8 \\
\hline He tenido frecuentemente sentimientos negativos... & 0.519 & 0.569 & 27.0 & 15.2 & 22.6 & 34.0 & 1.2 \\
\hline Desearía sentir más aprecio por mí mismo. & 0.520 & 0.364 & 38.4 & 20.0 & 13.0 & 27.2 & 1.4 \\
\hline
\end{tabular}

Dim. $=$ dimensión; Per. $=\%$ respuestas no respondidas.

Fuente: elaboración propia. 
Se realiza en primera instancia un análisis exploratorio con un método de extracción de componentes principales y con un método de rotación de normalización VARIMAX con Kaiser. Este análisis aporta una solución de cinco factores con autovalores superiores a 1 que explican el $60.49 \%$ de la varianza. Dado que la estructura teórica resultante de los análisis pilotos corresponde a cuatro factores, y dado el bajo aporte a la varianza de un quinto factor, se realiza un nuevo análisis solicitando al programa la extracción de cuatro factores, los cuales explican un $57.49 \%$ de la varianza (Tabla 2). La distribución de los factores fue similar a la propuesta teórica. El Factor 1 explica el $41.14 \%$ de la varianza, el 2 el $8.39 \%$, el 3 el $4.46 \%$ y el Factor 4 el $3.51 \%$ de la misma.

TABLA 2

Carga factorial, media y desviación típica de cada reactivo

\begin{tabular}{|c|c|c|c|c|c|c|}
\hline & 1 & 2 & 3 & 4 & media & DE \\
\hline La idea de quitarme la vida me viene repetidamente a la cabeza & 0.656 & 0.196 & 0.218 & 0.122 & 3.23 & 1.078 \\
\hline Creo que no merece la pena seguir viviendo & 0.598 & 0.277 & 0.197 & 0.039 & 3.39 & 0.995 \\
\hline Algunas veces me he imaginado a mi mismo sin vida & 0.542 & 0.168 & 0.068 & 0.241 & 3.00 & 1.155 \\
\hline He pensado en mi propio suicidio & 0.737 & 0.266 & 0.147 & 0.121 & 3.30 & 1.045 \\
\hline Le he comentado a mis amigos que me gustaría quitarme la vida & 0.602 & 0.199 & -0.142 & 0.259 & 3.52 & 0.937 \\
\hline Si me quisiera suicidar, tengo claro cómo lo haría & 0.661 & 0.183 & 0.064 & 0.074 & 3.35 & 1.084 \\
\hline He simulado mentalmente mi suicidio & 0.736 & 0.147 & 0.184 & 0.093 & 3.37 & 1.008 \\
\hline Se con claridad cómo poder suicidarme sin fallar & 0.680 & 0.149 & 0.077 & 0.081 & 3.42 & 1.025 \\
\hline Le he dicho a alguien que ya no quiero seguir viviendo & 0.678 & 0.182 & -0.010 & 0.221 & 3.35 & 1.068 \\
\hline Tengo planificada la forma de cómo podría quitarme la vida & 0.741 & 0.205 & 0.148 & 0.073 & 3.57 & 0.872 \\
\hline A veces he tenido la fantasía de verme ahorcado & 0.742 & 0.096 & 0.122 & 0.021 & 3.43 & 1.008 \\
\hline No se cómo, pero me quiero quitar la vida & 0.772 & 0.115 & 0.315 & 0.105 & 3.56 & 0.869 \\
\hline $\begin{array}{l}\text { A veces pienso en suicidarme de cualquier manera, ahorcándome, } \\
\text { quemándome o cualquier otra forma }\end{array}$ & 0.806 & 0.122 & 0.215 & 0.077 & 3.57 & 0.895 \\
\hline Quisiera morirme & 0.743 & 0.233 & 0.277 & 0.126 & 3.45 & 0.976 \\
\hline Siento que la vida tal como la vivo no vale la pena & 0.593 & 0.358 & 0.256 & 0.264 & 3.25 & 1.042 \\
\hline A veces me gustaría estar muerto y lejos de todo & 0.687 & 0.238 & 0.088 & 0.290 & 3.14 & 1.161 \\
\hline Estoy aburrido de vivir & 0.603 & 0.300 & 0.122 & 0.160 & 3.26 & 1.03 \\
\hline En general, estoy satisfecho conmigo mismo & 0.106 & 0.515 & 0.385 & 0.291 & 2.81 & 0.936 \\
\hline Me siento orgulloso/a de quien soy y la vida que llevo & 0.267 & 0.713 & 0.318 & 0.182 & 3.03 & 0.955 \\
\hline Estoy contento conmigo mismo & 0.271 & 0.687 & 0.340 & 0.220 & 2.96 & 0.951 \\
\hline Estoy conforme con mi vida actual & 0.331 & 0.746 & 0.233 & 0.137 & 2.88 & 0.992 \\
\hline Las condiciones de mi vida son excelentes & 0.266 & 0.729 & 0.045 & 0.080 & 2.59 & 1.008 \\
\hline Estoy satisfecho con mi vida & 0.323 & 0.755 & 0.163 & 0.197 & 2.90 & 1.018 \\
\hline Hasta el momento, he conseguido las cosas & 0.204 & 0.728 & 0.212 & 0.077 & 2.75 & 1.002 \\
\hline
\end{tabular}




\begin{tabular}{lcccccc}
\hline & 1 & 2 & 3 & 4 & media & DE \\
\hline Si pudiera volver a vivir mi vida, no cambiaría casi nada & 0.168 & 0.655 & 0.173 & 0.108 & 2.40 & 1.149 \\
Creo que soy importante para otros & 0.148 & 0.095 & 0.651 & 0.303 & 3.09 & 0.916 \\
Creo que tengo muchos motivos para enorgullecerme & 0.097 & 0.253 & 0.625 & 0.250 & 3.00 & 0.914 \\
Tengo claro qué es lo que intento conseguir en la vida & 0.250 & 0.277 & 0.641 & -0.010 & 3.17 & 0.921 \\
Tengo gente a mi alrededor que entiende mis puntos & 0.132 & 0.379 & 0.546 & -0.081 & 2.94 & 0.993 \\
Tengo clara la dirección y el objetivo de mi vida & 0.286 & 0.333 & 0.577 & -0.010 & 3.00 & 0.951 \\
A veces pienso que no sirvo para nada & 0.328 & 0.280 & 0.071 & 0.584 & 2.66 & 1.191 \\
Me he sentido solo & 0.210 & 0.060 & 0.037 & 0.722 & 2.20 & 1.159 \\
Me inclino a pensar que, en conjunto, soy un fracaso & 0.387 & 0.338 & 0.279 & 0.389 & 3.22 & 1.023 \\
He tenido frecuentemente sentimientos negativos, tales & 0.438 & 0.133 & 0.184 & 0.483 & 2.64 & 1.211 \\
Desearía sentir más aprecio por mí mismo & 0.036 & 0.175 & 0.128 & 0.727 & 2.29 & 1.242 \\
\hline
\end{tabular}

Fuente: elaboración propia.

Dados los requerimientos del programa Lisrel 8.3, el análisis factorial confirmatorio fue realizado sobre la base de aquellos cuestionarios donde fueron contestadas el $100 \%$ de las preguntas. De los 500 cuestionarios, 359 (72\%) cumplieron el requisito de no contener ni una sola respuesta perdida.

Los índices CFI (Comparative Fit Index), NFI (Normal Fit Index) y RFI (Relative Fit Index) muestran valores inferiores a 0.90 , valor considerado como de buen ajuste o superior (Bentler \& Dudgeon, 1996), sin embargo, se acercan a este. El índice RMSEA (Root Mean Square Error of Approximation) no resulta significativo al obtener un valor superior a 0.08 (Browne \& Cudeck, 1993). Estos indicadores, en conjunto con el índice de bondad de ajuste GFI, que representa el grado de ajuste conjunto (valores entre $0=$ mal ajuste, a 1 = ajuste perfecto), indican que el ajuste de los datos a la estructura factorial es moderado (Tabla 3).
La Tabla 4 muestra las correlaciones existentes entre las dimensiones y de las dimensiones con la escala total. Tal como se puede observar, estas son moderadas entre las distintas dimensiones, lo que permite suponer la independencia de las dimensiones, pero son altas al correlacionarlas con la escala total, lo que expresa la fuerte relación de cada una de ellas con la evaluación global de la escala.

\section{Capacidad discriminante}

Al consultar a los participantes sobre si habían intentado suicidarse, 30 de ellos $(6 \%)$ respondieron que sí, 462 que no $(92.4 \%)$ y 8 no respondieron (1.6\%). De los 30 que contestaron afirmativamente, 23 fueron mujeres $(77 \%)$ y 7 hombres $(23 \%)$.

La Tabla 5 contiene las medias obtenidas para cada una de las dimensiones y para la escala total, separadas entre quienes han intentado o no sui-

TABLA 3

Índices de ajuste para modelos factorial de cuatro dimensiones

\begin{tabular}{ccccccc}
\hline Escala & $\chi^{2}$ & CFI & NFI & GFI & RFI & RMSEA \\
\hline 4 factores & 2271.307 & 0.767 & 0.715 & 0.645 & 0.694 & 0.121 \\
\hline
\end{tabular}

$\chi^{2}=$ Normal Theory Weighted Least Squares Chi-Square; CFI = Comparative Fit Index; NFI = Normed Fit Index; GFI; Goodness of Fit Index; RFI = Relative Fit Index; RMSEA = Root Mean Square Error of Approximation.

Fuente: elaboración propia. 
TABLA 4

Correlaciones interdimensiones y dimensiones-escala total

\begin{tabular}{|c|c|c|c|c|c|}
\hline & & Satisfacción & Sentido de Vida & Afecto Negativo & Escala total \\
\hline \multirow{3}{*}{ Conductas Suicida } & $r$ & $0.624 * *$ & $0.509 * *$ & $0.633 * *$ & $0.829 * *$ \\
\hline & $p$ & 0.000 & 0.000 & 0.000 & 0.000 \\
\hline & $\mathrm{N}$ & 399 & 399 & 400 & 359 \\
\hline \multirow{3}{*}{ Satisfacción } & $r$ & & $0.642 * *$ & $0.586^{* *}$ & $0.865 * *$ \\
\hline & $p$ & & 0.000 & 0.000 & 0.000 \\
\hline & $N$ & & 436 & 435 & 359 \\
\hline \multirow{3}{*}{ Sentido de Vida } & $r$ & & & $0.447 * *$ & $0.788 * *$ \\
\hline & $p$ & & & 0.000 & 0.000 \\
\hline & $N$ & & & 442 & 359 \\
\hline \multirow{3}{*}{ Afecto Negativo } & $r$ & & & & $0.814 * *$ \\
\hline & $p$ & & & & 0.000 \\
\hline & $N$ & & & & 359 \\
\hline
\end{tabular}

$r=$ correlación de Pearson; $p=$ significación (bilateral); ** La correlación es significativa al nivel 0,01 (bilateral).

Fuente: elaboración propia.

TABLA 5

Medias de dimensiones Escala Riesgo Suicida según intento de suicidio

\begin{tabular}{lccccccccc}
\hline & \multicolumn{3}{c}{ Sin intento suicida } & \multicolumn{3}{c}{ Con intento suicida } & \multicolumn{3}{c}{ Muestra Total } \\
\cline { 2 - 10 } & $N$ & Media & $D E$ & $N$ & Media & DE & $N$ & Media & DE \\
\hline Conducta Suicida & 396 & 3.43 & 0.687 & 25 & 2.32 & 0.925 & 425 & 3.37 & 0.747 \\
Afecto Negativo & 432 & 2.68 & 0.821 & 30 & 1.76 & 0.758 & 468 & 2.62 & 0.848 \\
Satisfacción & 430 & 2.84 & 0.762 & 26 & 2.20 & 0.921 & 463 & 2.80 & 0.784 \\
Sentido de Vida & 437 & 3.06 & 0.633 & 27 & 2.80 & 0.852 & 468 & 3.05 & 0.649 \\
Escala Total & 339 & 3.03 & 0.583 & 19 & 2.31 & 0.861 & 359 & 2.99 & 0.619 \\
\hline
\end{tabular}

Fuente: elaboración propia.

cidarse. Las diferencias entre medias son estadísticamente significativas en todas las dimensiones: Conducta Suicida $(t(419)=7.669 ; p<0.001)$, Satisfacción $(t(454)=4.077 ; p<0.001)$; Sentido de Vida $(t(462)=2.046 ; p<0.05)$; Afecto Negativo $(t(460)=5.979 ; p<0.001)$ y para la escala total $(t(356)=5.077 ; p<0.001)$.
Análisis de regresión

La Tabla 6 entrega el nivel de significación de cada una de las variables independientes, además de los pesos relativos de cada una de las variables dentro del modelo de regresión 


\section{TABLA 6}

Coeficientes del modelo para variable a predecir "he intentado quitarme la vida" y variables predictoras: (Constante), Conductas Suicidas, Afecto Negativo y Satisfacción

\begin{tabular}{|c|c|c|c|c|c|c|}
\hline \multicolumn{2}{|c|}{ Modelo } & \multicolumn{2}{|c|}{$\begin{array}{l}\text { Coeficientes no } \\
\text { estandarizados }\end{array}$} & \multirow{2}{*}{$\begin{array}{c}\begin{array}{c}\text { Coeficientes } \\
\text { estandarizados }\end{array} \\
\text { Beta }\end{array}$} & \multirow[t]{2}{*}{$t$} & \multirow[t]{2}{*}{ Sig. } \\
\hline & & B & Error típ. & & & \\
\hline \multirow{5}{*}{ Total } & (Constante) & 3.355 & 0.191 & & 17.560 & 0.000 \\
\hline & Conductas Suicidas & -0.594 & 0.069 & -0.543 & -8.649 & 0.000 \\
\hline & Satisfacción & 0.065 & 0.066 & 0.067 & 0.982 & 0.327 \\
\hline & Sentido de Vida & -0.057 & 0.070 & -0.050 & -0.810 & 0.418 \\
\hline & Afecto negativo & -0.015 & 0.056 & -0.016 & -0.269 & 0.788 \\
\hline
\end{tabular}

Variable dependiente: "He intentado quitarme la vida".

Fuente: elaboración propia.

El modelo con las cuatro dimensiones explica el $29.2 \%$ de la varianza de la variable intento de suicidio. Como se puede observar en la última columna, solo la dimensión Conducta Suicida tiene una significación asociada inferior a 0.001 , teniendo una correlación lineal significativa con la variable intento de suicidio, contribuyendo de forma significativa al modelo.

Los coeficientes Beta estandarizados muestran los pesos relativos de cada una de las variables en el modelo de regresión. Cuanto mayor sea el valor del coeficiente, mayor es el peso de esa variable en el modelo. Como se puede ver, la dimensión que posee peso relativo mayor dentro del modelo de regresión es la dimensión Conducta Suicida (0.543).

\section{Discusión}

A partir de los datos reportados se deduce que los intentos de suicidio son similares a los encontrados en otros estudios sudamericanos (Botega, Azevedo, de Oliveira, Dalgalarrondo \& Marín, 2005), siendo igualmente mayor el porcentaje de mujeres que han cometido un intento de suicidio en relación con el de los hombres, tal como es reportado sistemáticamente en la literatura.

Los porcentajes de ideación suicida son similares al valor superior del rango reportado por Haquin et al. (Haquin, Larraguibel \& Cabezas,
2004) en una muestra de edades similares, probablemente, debido al tipo de preguntas realizadas, siendo de todas maneras sensiblemente menor que el reportado en otros estudios en adolescentes (Roxo \& Guevara, 2006).

Se sugiere seguir evaluando las propiedades del instrumento, a fin de evaluar su comportamiento psicométrico en otras poblaciones en riesgo, tales como adolescentes no escolarizados o muestras clínicas, quienes presentan una mayor vulnerabilidad.

\section{Referencias}

Apter, A., Gothelf, D., Orbach, Y., Weizman, R., Ratzoni, G., Har-Even, D. et al. (1995). Correlation of suicidal and violent behavior in different diagnostic categories in hospitalized adolescent patients. J. Am. Acad. Child Adolesc. Psychiatry, 34, 912-918.

Bentler, M. P. \& Dudgeon, P. (1996). Covariance structure analysis: Statistical practice, theory and directions. Annual Review of Psychology, 47, 563-592.

Birmaher, B., Ryan, N. D. \& Williamson, D. E. (1996). Childhood and adolescent depression: A review of the past 10 years, Part I.J. Am. Acad. Child Adolesc. Psychiatry, 35, 1427-1439.

Bolton, M. J., Cox, P. B., Afifi, M. T., Enns, M. M., Bienvenu, M. P. \& Sareen, M. J. (2008). Anxiety disorders and risk for suicide attempts: Findings 
from the Baltimore epidemiologic catchment area follow-up study. Depression and Anxiety, 25, 477 481.

Botega, N., Berti de Azevedo, M., Bosco de Oliveira, H., Dalgalarrondo, P. \& Marín León, L. (2005). Suicidal behavior in the community: Prevalence and factor associated with suicidal ideation. Rev. Bras. Psiquiatr., 27, 45-53.

Brent, D. A., Perper, J. A. \& Moritz, G. (1993). Psychiatric risk factors for adolescent suicide: A case-control study. J. Am. Acad. Child. Adolesc. Psychiatry, 32, 521-529.

Browne, M. W. \& Cudeck, R. (1993). Alternative ways of assessing model fit. In K. A. Bollen \& J. S. Long (Eds.), Testing structural equation models (pp. 136162). Beverly Hills, CA: Sage.

Cendales, R., Vanegas, C., Fierro, M., Córdoba, R. \& Olarte, A. (2007). Tendencias del suicidio en Colombia, 1985-2002. Rev. Panam. Salud Pública, 22, 231-238.

Fleming, T. M. (2007). Self-reported suicide attempts and associated risk and protective factors among secondary school students in New Zealand. Australian and New Zealand Journal of Psychiatry, 41 (3), 213-221.

Haquin, C., Larraguibel, M. \& Cabezas, J. (2004). Factores protectores y de riesgo en salud mental en niños y adolescentes de la ciudad de Calama. Rev. Child. Pediatr., 75, 425-433.

Harrington, R., Bredenkamp, D. \& Groothues, C. (1994). Adult outcomes of child and adolescent depression III. Links with suicidal behaviours. J. Child Psychol. Psychiatry, 35, 1309-1319.

Hawton, K. \& Fagg, J. (1992). Deliberate self-poisoning and self injury in adolescents: A study of characteristics and trends in Oxford, 1976-1989. Br. J. Psychiatry, 161, 816-823.

Hidaka, Y., Operario, D., Takenaka, M., Omori, S., Ichikawa, S. \& Shirasaka, T. (2008). Attempted suicide and associated risk factors among youth in urban Japan. Soc. Psychiatry Psychiatr. Epidemiol., 43, 752-757.

Hollis, C. (1996). Depression, family environment, and adolescent suicidal behavior. J. Am. Acad. Child Adolesc Psychiatry, 35, 622-630.
Krysinska, K. \& De Leo, D. (2008). Suicide on railway networks: Epidemiology, risk factors and prevention. Australian and New Zealand Journal of Psychiatry, 42 (9), 763-771.

Larraguibel, M., González, P., Martínez, V. \& Valenzuela, R. (2000). Factores de riesgo de la conducta suicida en niños y adolescentes. Rev. Chil. Pediatr., 71, 183-191.

Li, X. Y., Phillips, M. R., Zhang, Y. P., Xu, D. \& Yang, G. H. (2008). Risk factors for suicide in China's youth: A case-control study. Psychological Medicine, 38, 397-406.

Mayer, P. \& Ziaian, T. (2002). Suicide, gender, and age variations in India: Are women in Indian society protected for suicide? Crisis, 23, 98-103.

Minoletti, A. \& López, C. (1999). Enfermedades mentales en Chile. Magnitudes y consecuencias. Santiago: Departamento Programa de las Personas. Unidad de Salud Mental, Ministerio de Salud.

Ono, Y., Kawakami, N., Nakane, Y., Nakamura, Y., Tachimori, H., Iwata, N. et al. (2008). Prevalence of and risk factors for suicide-related outcomes in the World Health Organization World Mental Health Surveys Japan. Psychiatry and Clinical Neurosciences, 62 (4), 442-449.

Phillips, M., Li, X. \& Zhang, Y. (2002). Suicide rates in China. The Lancet, 359 (9325), 2274-2275.

Puentes-Rosas, E., López-Nieto, L. \& Martínez-Monroy, T. (2004). La mortalidad por suicidios: México 1990-2001. Rev. Panam. Salud Pública, 16, 102 109.

Roxo, V. \& Guevara, B. (2006). Estudo de ideacao suicida em adolescentes de 15 a 19 anos. Estudos de Psicologia, 11, 345-351.

Shaffer, D., Gould, M. S. \& Fisher, P. (1996). Psychiatric diagnosis in child and adolescent suicide. Arch. Gen. Psychiatry, 53, 339-348.

Shur-Fen Gau, S., Chen, Y., Tsai, F., Lee, M., Chiu, Y., Soong, W. \& Hwu, H. (2008). Risk factors for suicide in Taiwanese college students. Journal of American College Health, 57, 135-142.

Silvikena, A. \& Kvernmob, S. (2007). Suicide attempts among indigenous Sami adolescents and majority peers in Arctic Norway: Prevalence and associated risk factors. Journal of Adolescence, 30, 613-626. 
Soo Kim, H. \& Sil Kim, H. (2008). Risk factors for suicide attempts among Korean adolescents. Child Psychiatry Hum. Dev., 39, 221-235.

Randell, B., Wang, W-1., Herting, J. \& Eggert, L. (2006). Family factors predicting categories of suicide risk. Joumal of Child and Family Studies, 15 (3), 255-270.

Valdivia, M., Ebner, D., Fierro, V., Fajardo, C. \& Miranda, R. (2001). Hospitalización por intento de suicidio en población pediátrica: una revisión de cuatro años. Rev. Chil. Neuro-Psiquiatr., 3(3), 211-218.

Valtonen, H., Suominen, K., Partonen, T., Ostamo, A. \& Lönnqvist, J. (2006). Time patterns of attempted suicide. Journal of Affective Disorders, 90, 201-207.

Vijayakumar, L., John, S., Pirkis, J. \& Whiteford, H. (2005). Suicide in developing countries. Crisis, 26, 112-119.
Yunes, J. \& Zubarew, T. (1999). Mortalidad por causas violentas en adolescentes y jóvenes: un desafío para la región de las Américas. Rev. Bras. Epidemiol., 2, 102-171.

Yunes, J. \& Rajs, D. (1994). Tendencia de la mortalidad por causas violentas en la población general y entre los adolescentes y jóvenes de la región de las Américas. Cad. Saúde Públ., 10, 88-125.

World Health Organization. (2008). Suicide rates per 100.000 by country, year and sex. Recuperado el 26 de enero, 2008, de http://www.who.int/mental health/prevention/suicide_rates/en/index.html

Wong, J. \& Stewart, S. (2007). Risk factors associated with suicide attempts and other self-injury among Hong Kong adolescents. Suicide and Life-Threatening Behavior, 37 (4), 453-466. 


\section{Anexo 1}

Marco Conceptual Factores Escala Riesgo Suicida

\begin{tabular}{|c|c|c|c|}
\hline Factor & Componente & Descripción & Preguntas \\
\hline \multirow{17}{*}{$\begin{array}{l}\text { Conductas } \\
\text { Suicidas }\end{array}$} & \multirow{5}{*}{ Deseo de morir } & \multirow{5}{*}{$\begin{array}{l}\text { Inconformidad e insatisfac- } \\
\text { ción de la persona con su } \\
\text { vida presente }\end{array}$} & Creo que no merece la pena seguir viviendo \\
\hline & & & Quisiera morirme \\
\hline & & & A veces me gustaría estar muerto y lejos de todo \\
\hline & & & Siento que la vida tal como la vivo no vale la pena \\
\hline & & & Estoy aburrido de vivir \\
\hline & \multirow{2}{*}{$\begin{array}{l}\text { Representación } \\
\text { suicida }\end{array}$} & \multirow{2}{*}{$\begin{array}{l}\text { Imágenes mentales del sui- } \\
\text { cidio del propio individuo }\end{array}$} & He pensado en mi propio suicidio \\
\hline & & & Algunas veces me he imaginado a mi mismo sin vida \\
\hline & \multirow{8}{*}{ Ideas suicidas } & \multirow{8}{*}{$\begin{array}{l}\text { Pensamientos de terminar } \\
\text { con la propia existencia, } \\
\text { puede ser sin un método } \\
\text { específico, con un método } \\
\text { inespecífico, con un método } \\
\text { específico no planificado o } \\
\text { bien una idea suicida pla- } \\
\text { nificada }\end{array}$} & $\begin{array}{l}\text { A veces he tenido la fantasía de verme ahorcado o } \\
\text { muerto de alguna otra manera }\end{array}$ \\
\hline & & & No sé cómo, pero me quiero quitar la vida \\
\hline & & & $\begin{array}{l}\text { A veces pienso en suicidarme de cualquier manera, } \\
\text { ahorcándome, quemándome o cualquier otra forma }\end{array}$ \\
\hline & & & Si me quisiera suicidar, tengo claro cómo lo haría \\
\hline & & & $\begin{array}{l}\text { La idea de quitarme la vida me viene repetidamente a } \\
\text { la cabeza }\end{array}$ \\
\hline & & & $\begin{array}{l}\text { Tengo planificada la forma de cómo podría quitarme } \\
\text { la vida }\end{array}$ \\
\hline & & & He simulado mentalmente mi suicidio \\
\hline & & & Se con claridad cómo poder suicidarme sin fallar \\
\hline & \multirow[t]{2}{*}{$\begin{array}{l}\text { Amena- } \\
\text { za suicida }\end{array}$} & \multirow{2}{*}{$\begin{array}{l}\text { Insinuación o afirmación } \\
\text { verbal de las intenciones } \\
\text { suicidas ante personas que } \\
\text { puedan impedirlo }\end{array}$} & $\begin{array}{l}\text { Le he comentado a mis amigos que me gustaría qui- } \\
\text { tarme la vida }\end{array}$ \\
\hline & & & Le he dicho a alguien que ya no quiero seguir viviendo \\
\hline $\begin{array}{l}\text { Intento } \\
\text { suicida }\end{array}$ & \multicolumn{2}{|c|}{$\begin{array}{l}\text { Tentativa de suicidio, intento de autoelimi- } \\
\text { nación o autolesión intencionada. Acto en el } \\
\text { cual un individuo deliberadamente se hace } \\
\text { daño a sí mismo }\end{array}$} & He intentado quitarme la vida \\
\hline
\end{tabular}

Me siento orgulloso/a de quien soy y la vida que llevo

Satisfacción Consigo mismo

Estoy contento conmigo mismo

En general, estoy satisfecho conmigo mismo 


\begin{tabular}{|c|c|c|c|}
\hline Factor & Componente & Descripción & Preguntas \\
\hline \multirow{5}{*}{ Satisfacción } & \multirow{5}{*}{ Con la Vida } & & Las condiciones de mi vida son excelentes \\
\hline & & & Estoy conforme con mi vida actual \\
\hline & & & Estoy satisfecho con mi vida \\
\hline & & & $\begin{array}{l}\text { Hasta el momento, he conseguido las cosas importan- } \\
\text { tes que quiero en la vida }\end{array}$ \\
\hline & & & $\begin{array}{l}\text { Si pudiera volver a vivir mi vida, no cambiaría casi } \\
\text { nada }\end{array}$ \\
\hline \multirow{6}{*}{$\begin{array}{l}\text { Afectos } \\
\text { negativos }\end{array}$} & & & Me he sentido solo \\
\hline & & & $\begin{array}{l}\text { He tenido frecuentemente sentimientos negativos, } \\
\text { tales }\end{array}$ \\
\hline & & & como tristeza, desesperanza, ansiedad, depresión \\
\hline & & & Desearía sentir más aprecio por mí mismo \\
\hline & & & A veces pienso que no sirvo para nada \\
\hline & & & Me inclino a pensar que, en conjunto, soy un fracaso \\
\hline \multirow{5}{*}{$\begin{array}{l}\text { Sentido de } \\
\text { vida }\end{array}$} & A utoestima & & Creo que soy importante para otros \\
\hline & 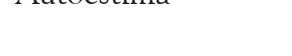 & & Creo que tengo muchos motivos para enorgullecerme \\
\hline & \multirow{3}{*}{ Proyección al futuro } & & Tengo claro qué es lo que intento conseguir en la vida \\
\hline & & & Tengo gente a mi alrededor que entiende mis puntos \\
\hline & & & Tengo clara la dirección y el objetivo de mi vida \\
\hline
\end{tabular}

